\title{
Modelo de protocolo experimental para induzir, classificar e avaliar as enterites inespecíficas em frangos de corte ${ }^{1}$
}

\author{
Dany Mesa², Mariana Lourenço², Patrick Westphal ${ }^{2}$, Antonio Kraieski² \\ e Elizabeth Santin ${ }^{2 *}$
}

\begin{abstract}
Mesa D., Lourenço M., Westphal P., Kraieski A. \& Santin E. 2014. [Experimental protocol model to induce, classified and evaluate non specific enteritis in broilers.] Modelo de protocolo experimental para induzir, classificar e avaliar as enterites inespecíficas em frangos de corte. Pesquisa Veterinária Brasileira 34(10):929-936. Laboratório de Microbiologia e Ornitopatologia, Departamento de Medicina Veterinária, Universidade Federal do Paraná, Rua dos Funcionários 1540, Bairro Juvevê, Curitiba, PR 80035-050, Brazil. E-mail: santin@ufpr.br

Several negative conditions affect broilers intestinal health and reduce their performance. Feed additives, called growth promoters, are used in broilers production to control enteric problems, but is very difficult to evaluate these products in experimental conditions. The aim of this study was to evaluate the best experimental model to induce enteritis in broilers. A 192 one-day-old male broilers (Cobb 500 ${ }^{\circledR}$ ) were divided into $2 \times 4$ a factorial design (with good or poor quality oil in diet and with or without coccidiosis and Gumboro disease vaccine), with eight treatments. The birds were housed on litter, with water and feed ad libitum and were weighed weekly. At days 14, 21, 28 and 35, six birds per treatment were euthanized and gut gross lesions evaluated. Samples of duodenum, jejunum and ileum were taken for histopathological evaluation. A standard lesion score considering lymphocytic infiltration, enterocytes morphology, interstitial edema, and lymph vessel dilation was used. It was observed that broilers fed with poor quality of oil in the diet showed lower body weight and more severe macroscopic and histopathologic lesions on all intestinal segments. These lesions were more severe in birds challenged with coccidiosis and Gumboro disease. The results suggest that an inclusion of poor quality of oil in diet, associated with challenge with coccidiosis at first day and Gumboro virus vaccine at 16 day is the best protocol to promote enteritis in broilers at experimental conditions, and that use of standard lesion score was useful to evaluate enteritis.
\end{abstract}

INDEX TERMS: Enteritis, experimental protocol, standard score of lesions, histopathology, broilers.

RESUMO.- Vários fatores negativos podem afetar a saúde intestinal de frangos de corte e reduzir o seu desempenho. Aditivos para alimentação animal, chamados melhoradores de crescimento são utilizados na produção de frangos para controlar os problemas intestinais. Entretanto, a dificuldade de se induzir enterites em condições experimentais torna difícil a avaliação destes produtos. 0 objetivo deste estudo foi avaliar o melhor modelo experimental para in-

\footnotetext{
${ }^{1}$ Recebido em 14 de outubro de 2013.

Aceito para publicação em 11 de janeiro de 2014.

2 Laboratório de Microbiologia e Ornitopatologia, Departamento de Medicina Veterinária, Universidade Federal do Paraná (UFPR), Rua dos Funcionários 1540, Curitiba, PR 80035-050, Brasil. *Autor para correspondência: santin@ufpr.br
}

duzir enterite em frangos de corte. Foram utilizados 192 pintinhos de corte, machos (Cobb $500^{\circledR}$ ), divididos em esquema fatorial $2 \times 4$ (com boa ou baixa qualidade do óleo na dieta e com ou sem vacina contra coccidiose e doença de Gumboro), com oito tratamentos. As aves foram alojadas em cama de maravalha, com água e ração à vontade, e foram pesadas semanalmente. Nos dias 14, 21, 28 e 35, seis aves por tratamento foram abatidas para avaliação de lesões macroscópicas e microscópicas mediante a implementação de um sistema padrão de classificação de severidade das lesões que considerou infiltração linfocítica, morfologia dos enterócitos, edema intersticial e dilatação dos vasos linfáticos na mucosa do intestino. Foi observado que frangos alimentados com gordura de baixa qualidade na ração 
apresentaram menor ganho de peso e maior severidade de lesões histológicas em todos os segmentos intestinais. Estas lesões foram mais severas em aves desafiadas com coccidiose e doença de Gumboro. Estes resultados sugerem que a inclusão de gordura de baixa qualidade na ração, associada ao desafio com cocciciose no primeiro dia de vida e contra doença de Gumboro no 16을 dia, é melhor protocolo para induzir enterite em frangos de corte em condições experimentais, e ainda que o sistema padrão de classificação de severidade de lesões intestinais foi adequado para avaliar as enterites em frangos de corte.

TERMOS DE INDEXAÇÃO: Enterites, protocolo experimental, padronização de escore de lesões, histopatologia, frangos de corte.

\section{INTRODUÇÃO}

$\mathrm{Na}$ indústria avícola, o melhoramento em genética, nutrição, saúde (biosseguridade) e bem-estar foram cruciais para melhorar a produtividade (Simitzis et al. 2012). Associados, esses fatores possibilitaram que a taxa de crescimento média atual de frangos de corte seja de 2,83 gramas por hora (g/h), enquanto que em 1990 era de apenas 1,87g/h (Patricio et al. 2012). De acordo com Havenstein et al. (2003), nos últimos 44 anos, o ciclo de crescimento do frango diminuiu para um terço do tempo (de 101 dias em 1957 para 32 dias em 2001), diminuindo em três vezes o consumo de alimento. Em termos de eficiência, a conversão alimentar do frango de corte é melhor dentre todos os sistemas de produção de carne, ou seja, enquanto para frangos este índice encontra-se em 1,69, para suínos e bovinos é de 2,99 e 10,4, respectivamente (Tolkamp 2010).

Porém, existem ainda várias condições negativas que podem resultar em problemas entéricos em frangos de corte, que afetam a digestão de nutrientes da dieta, o equilíbrio da microbiota intestinal e, consequentemente, os parâmetros de produção (Zekarias et al. 2005, Teirlynck et al. 2011).

Dentre estas condições negativas encontram-se os agentes infecciosos e não infecciosos. Os principais agentes infecciosos são Eimeria spp. e Clostridium perfringens (Williams 2005, Timbermont et al. 2011), que normalmente associam-se a fatores não infecciosos, como mudanças ou interrupção na alimentação, desequilíbrio nutricional, polissacarídeos não amiláceos, gordura de baixa qualidade, disfunção enzimática e micotoxinas (Hetland et al. 2004, Dänicke et al. 2007, Teirlynck et al. 2009). Assim, a maioria dos quadros clínicos entéricos observados em frangos de corte no campo tem a causa primária desconhecida.

Além dos fatores mencionados que afetam diretamente a saúde do intestino das aves, pode-se ainda citar doenças que causam imunossupressão, como a doença de Gumboro e a doença de Marek (Hoerr 2010), entre outras, como fatores predisponentes para as enterites subclínicas. Na doença de Gumboro, o uso de vacinas fabricadas com estirpes de baixa ou intermediária atenuação também podem afetar, de forma transitória, a resposta imunológica da ave e a resposta a agentes entéricos (Mazariegos et al. 1990).

Nos últimos anos, poucas pesquisas têm-se focado nas diferentes formas de controle deste tipo de enterite (denominada inespecífica), e no entendimento de sua pato- gênese. Isso provavelmente está associado à dificuldade de reproduzir o quadro sob condições experimentais, de estabelecer padrões de severidade e de classificar as lesões (Olkowski et al. 2008) que permitam avaliar o efeito de componentes nutricionais (McReynolds et al. 2004, Lokaewmanee et al. 2012), aditivos alimentares (Lee et al. 2010, Jayaraman et al. 2013), bem como o uso de antimicrobianos (Lanckriet et al. 2010, Rambozzi et al. 2012) e vacinas (Jang et al. 2012, Awad et al. 2013) na indução ou no controle destas enterites.

Neste cenário, o objetivo do presente trabalho foi estudar e comparar a associação de fatores nutricionais e infecciosos na capacidade de induzir enterite em frangos de corte, bem como estabelecer escores de lesões intestinais que permitam a padronização destes para o estudo de enterites nesses animais.

\section{MATERIAL E MÉTODOS}

Instalações, animais e dietas. Foram alojados 192 frangos de corte de um dia de idade, machos (Cobb $500^{\circledR}$ ), divididos em esquema fatorial 2 (com e sem gordura de baixa qualidade na dieta) x 4 (com e sem desafio de Eimeria e vacina com uma estirpe de atenuação intemediária do vírus da doença de Gumboro), com 8 tratamentos de 24 pintinhos cada, considerando cada ave como uma unidade experimental (Quadro 1).

No início do experimento, as aves foram pesadas, identificadas com anilhas na asa e distribuídas uniformemente de acordo com o peso inicial entre os tratamentos. As aves foram alojadas em salas com pressão negativa, previamente limpas e desinfetadas, em cama de maravalha esterilizada em autoclave a $121^{\circ} \mathrm{C}$ por 15 minutos.

A gordura utilizada foi proveniente de restos de fritura industrial, que de acordo com as análises laboratoriais continha uma concentração de peróxido de 6 meq $2 \mathrm{~kg}^{-1}$, contra 1,7 meq $2 \mathrm{~kg}^{-1} \mathrm{de}$ peróxido do óleo de boa qualidade. As rações foram formuladas de acordo com Rostagno (2011). Foram utilizados três tipos de ração, sendo ração inicial (1-21 dias), ração crescimento (22-35 dias) e ração final (36-42 dias) (Quadro 2). As amostras das matérias primas utilizadas nas rações (milho, farelo de soja e farelo de arroz) foram coletadas e enviadas ao Laboratório Samitec para a realização de análise de micotoxinas (aflatoxina, ocratoxina, fumonisina, DON e toxina T2) de acordo com a metodologia de cromatografia líquida de alta eficiência acoplada à espectrofotometria de massas.

Desafio vacinal. Os diferentes tratamentos utilizados foram classificados de acordo com o tipo de gordura na ração e com a utilização ou não de um protocolo de desafio vacinal com Eimeria (utilizando 10 vezes a dose recomendada pelo fabricante de uma vacina comercial contra à coccidiose) e/ou com a vacina co-

Quadro 1. Tratamentos do experimento

\begin{tabular}{|c|c|c|c|c|c|c|}
\hline & \multicolumn{2}{|c|}{ Gordura na ração } & \multicolumn{2}{|c|}{ Vacina coccidiose } & \multicolumn{2}{|c|}{ Vacina Gumboro } \\
\hline & $\begin{array}{c}\text { Boa } \\
\text { qualidade }\end{array}$ & $\begin{array}{c}\text { Baixa } \\
\text { Qualidade }\end{array}$ & Dia 1 & Dia 20 & Dia 16 & $\begin{array}{c}\text { Não } \\
\text { vacinado }\end{array}$ \\
\hline $\mathrm{T} 1$ & $\mathrm{x}$ & & & & & $\mathrm{x}$ \\
\hline $\mathrm{T} 2$ & $\mathrm{x}$ & & $\mathrm{x}$ & & & $\mathrm{x}$ \\
\hline T3 & $\mathrm{x}$ & & $\mathrm{x}$ & & $\mathrm{X}$ & \\
\hline $\mathrm{T} 4$ & $\mathrm{x}$ & & & $\mathrm{x}$ & $\mathrm{X}$ & \\
\hline T5 & & $\mathrm{x}$ & & & & $\mathrm{x}$ \\
\hline T6 & & $\mathrm{x}$ & $\mathrm{x}$ & & & $\mathrm{x}$ \\
\hline $\mathrm{T} 7$ & & $x$ & $\mathrm{x}$ & & $\mathrm{X}$ & \\
\hline T8 & & $\mathrm{x}$ & & $\mathrm{x}$ & $\mathrm{X}$ & \\
\hline
\end{tabular}


Quadro 2. Ingredientes e composição das dietas

\begin{tabular}{lcccccc}
\hline Matéria-prima (\%) & \multicolumn{2}{r}{$\begin{array}{r}\text { ração inicial } \\
(1-21 \text { dias })\end{array}$} & \multicolumn{2}{c}{ ração crescimento } \\
& (22-35 dias) & \multicolumn{2}{c}{$\begin{array}{c}\text { Final } \\
\text { (36-42d) }\end{array}$} \\
& T1-T4 & T5-T8 & T1-T4 & T5-T8 & T1-T4 & T5-T8 \\
\hline Milho & 87,682 & 87,682 & 81,015 & 81,015 & 20,939 & 20,939 \\
Farelo de soja & 56,819 & 56,819 & 41,889 & 41,889 & 8,696 & 8,696 \\
Gordura boa qualidade & 2,688 & 0,000 & 4,211 & 0,000 & 1,190 & 0,000 \\
Gordura baixa qualidade & 0,000 & 2,688 & 0,000 & 4,210 & 0,000 & 1,190 \\
Farelo de arroz & 4,800 & 4,800 & 8,400 & 8,400 & 3,150 & 3,150 \\
Fosfato dicalcico & 4,659 & 4,659 & 1,721 & 1,721 & 0,349 & 0,349 \\
Carbonato de cálcio & 1,809 & 1,809 & 1,204 & 1,204 & 0,278 & 0,278 \\
L-Lisina & 0,262 & 0,262 & 0,339 & 0,339 & 0,105 & 0,105 \\
Sal & 0,568 & 0,568 & 0,540 & 0,540 & 0,115 & 0,115 \\
L-Treonina & 0,065 & 0,065 & 0,092 & 0,092 & 0,030 & 0,030 \\
DL-Metionina & 0,372 & 0,372 & 0,348 & 0,348 & 0,084 & 0,084 \\
Premix vitaminas* & 0,192 & 0,192 & 0,168 & 0,168 & 0,042 & 0,042 \\
Premix minerais** & 0,080 & 0,080 & 0,070 & 0,070 & 0,017 & 0,017
\end{tabular}

Composição do premix: *Vitamina A (262500 UI/kg); Vitamina D3 (52500 UI/kg); Vitamina E (450 UI/kg); Vitamina K3 (50mg/ $\mathrm{kg})$; Vitamina B1 (44mg/kg); Vitamina B2 (150mg/kg); Vitamina B6 $(90 \mathrm{mg} / \mathrm{kg})$; Vitamina B12 (360mcg/kg); Niacina $(900 \mathrm{mg} / \mathrm{kg})$; Colina $(9450 \mathrm{mg} / \mathrm{kg}) ;$ Pantotenato de cálcio $(300 \mathrm{mg} / \mathrm{kg}) ;$ Ácido fólico (22,5mg/kg); Biotina (1,5mg/kg). ${ }^{* *}$ Cálcio $(230$ a $240 \mathrm{~g} / \mathrm{kg})$; Fósforo (68 g/kg); Sódio (42g/kg); Ferro (3300mg/kg); Manganês $(1900 \mathrm{mg} /$ $\mathrm{kg}) ;$ Zinco $(1500 \mathrm{mg} / \mathrm{kg}) ;$ Cobre $(18 \mathrm{~g} / \mathrm{kg}) ;$ Iodo $(24 \mathrm{mg} / \mathrm{kg}) ;$ Selênio $(7 \mathrm{mg} / \mathrm{kg})$; Metionina $(45 \mathrm{~g} / \mathrm{kg})$.

mercial contra a doença de Gumboro (na dose recomendada pelo fabricante). 0 desafio com Eimeria correspondeu a 20.000 oocistos/ave de (Eimeria acervulina, E. maxima, E. tenella, E. necatrix e E. brunetti). A vacinação contra a doença de Gumboro correspondeu a uma concentração de $\geq 2.0 \log ^{10} \mathrm{DIE}_{50}$.

Amostras. Nos dias 14, 21, 28 e 35 foram eutanasiadas seis aves de cada tratamento para a avaliação de enterites ocasionadas pelos desafios adotados nos diferentes tratamentos. Nestes animais, foi realizada a avaliação de presença de lesão macroscópica nos diferentes segmentos do intestino delgado (duodeno, jejuno e íleo), bem como a coleta de amostras destes segmentos (duodeno, jejuno - proximal ao divertículo de Meckel e íleo - proximal à junção ileocecal) para exame histopatológico.

Para a realização das análises histopatológicas, os fragmentos coletados foram fixados em formol tamponado a $10 \%$ por no mínimo 24 horas e posteriormente preparados e corados com hematoxilina e eosina. Foram avaliados 20 campos por fragmento em aumento de 20 vezes em microscópio óptico (Olympus America INC., NY, USA), totalizando 120 avaliações por tratamento. Para a análise macroscópica e microscópica realizada nas necropsias de 14, 21, 28 e 35 dias, foi utilizada a metodologia de Bracarense et al. (2012) modificada, na qual, para cada alteração macroscópica (Quadro 3) e microscópica (Quadro 4) observada na mucosa intestinal das aves, foi atribuído um grau de severidade (GS) entre 0 e 3, sendo grau 0 sem alteração, grau 1 alteração leve, grau 2 alteração moderada e grau 3 alteração severa, de acordo com a sua importância em reduzir a capacidade funcional do órgão, ou seja, digestão e absorção de alimentos. Para cada alteração observada foram atribuídos escores de 0 a 3 conforme a intensidade da lesão, escore 0 sem lesão, escore 1 é baixa extensão (25\%), escore 2 é média extensão (50\%), e escore 3 é grande extensão (75\%). Os valores de escores foram multiplicados pelo GS, estabelecendo um valor total de alterações visualizadas na mucosa de cada segmento intestinal.

Análise estatística. Os dados obtidos foram submetidos ao teste de normalidade de Shapiro-Wilk. Nos dados paramétricos foi realizada análise fatorial $2 \times 4$ (dois tipos de ração e quatro tratamentos vacinais) pela análise de variância (ANOVA), com poste-
Quadro 3. Classificação das lesões macroscópicas observadas no intestino

\begin{tabular}{|c|c|c|c|c|}
\hline Órgão & Parâmetros & $\begin{array}{c}\text { Grau de severidade } \\
\qquad(0 \text { a } 3)\end{array}$ & $\begin{array}{c}\text { Intensidade } \\
\text { da lesão (0 a 3) }\end{array}$ & \\
\hline \multirow[t]{3}{*}{ Duodeno } & $\begin{array}{c}\text { Acúmulo de material } \\
\text { mucoide }\end{array}$ & 1 & 3 & 3 \\
\hline & Congestão & 2 & 3 & 6 \\
\hline & Petéquias & 2 & 3 & 6 \\
\hline \multirow[t]{3}{*}{ Jejuno } & $\begin{array}{l}\text { Acúmulo de material } \\
\text { mucoide }\end{array}$ & l & 3 & 3 \\
\hline & Congestão & 2 & 3 & 6 \\
\hline & Petéquias & 2 & 3 & 6 \\
\hline \multirow[t]{5}{*}{ Íleo } & $\begin{array}{c}\text { Acúmulo de material } \\
\text { mucoide }\end{array}$ & 1 & 3 & 3 \\
\hline & $\begin{array}{l}\text { Placas de Peyer } \\
\text { mais aumentadas }\end{array}$ & 1 & 3 & 3 \\
\hline & Congestão & 2 & 3 & 6 \\
\hline & Petéquias & 2 & 3 & 6 \\
\hline & Ração não digerida & 2 & 3 & 6 \\
\hline Total & & & & 54 \\
\hline
\end{tabular}

* Modificado de Bracarense et al. (2012).

Quadro 4. Classificação das lesões microscópicas observadas na mucosa intestinal do duodeno, jejuno e íleo

\begin{tabular}{lccc}
\hline Parâmetros & $\begin{array}{c}\text { Grau de } \\
\text { severidade (0 a 3) }\end{array}$ & $\begin{array}{c}\text { Intensidade } \\
\text { da lesão (0 a 3) }\end{array}$ & \\
\hline $\begin{array}{l}\text { Dilatação de vasos linfáticos } \\
\text { Vacuolização citoplasmática }\end{array}$ & 1 & 3 & 3 \\
$\begin{array}{l}\text { de enterócitos } \\
\text { Infiltração de linfócitos na }\end{array}$ & 2 & 3 & 3 \\
lâmina própria & & 3 & 6 \\
$\begin{array}{l}\text { Dilatação de vilosidades } \\
\text { Fusão de vilosidades }\end{array}$ & 2 & 3 & 6 \\
$\begin{array}{l}\text { Edema intersticial de vilosidade } \\
\text { Hiperplasia de células caliciformes }\end{array}$ & 2 & 3 & 6 \\
$\begin{array}{l}\text { Presença de plasmócitos na } \\
\text { lâmina própria }\end{array}$ & 2 & 3 & 6 \\
Necrose apical de vilosidade & 3 & 3 & 6 \\
Total & & 3 & 6 \\
\end{tabular}

* Modificado de Bracarense et al. (2012).

rior teste de Tukey $(\mathrm{P}<0,05)$ quando as médias apresentaram diferença significativa. Dados não paramétricos foram submetidos ao teste de Kruskal-Wallis $(\mathrm{P}<0,05)$ considerando os fatores ração e tratamentos vacinais, quando não existiu diferença para este parâmetro foram analisados os tratamentos vacinais em separado.

\section{RESULTADOS}

\section{Micotoxinas}

A amostra de milho apresentou 1.090ppb e 418ppb de fumonisina B1 e fumonisina B2, respectivamente. O farelo de soja apresentou 148ppb de fumonisina B1. 0 farelo de arroz foi negativo para todas as micotoxinas avaliadas.

\section{Ganho de peso}

Em todo o período de vida das aves não foi observado o efeito da qualidade da gordura sobre o ganho de peso. Entre o período de 14-28 dias foi observado que os frangos desafiados com coccidiose no primeiro dia de vida e vacinados contra a doença de Gumboro no dia 16 apresentaram baixo ganho de peso quando comparado com o grupo controle, que não recebeu vacina ou desafio (Quadro 5). Entretan- 
to, quando se avaliou o período total do experimento (1-35 dias), não se observou efeito nem da qualidade de gordura utilizada, nem dos desafios vacinais sobre o desempenho das aves.

\section{Lesões macroscópicas}

As principais lesões macroscópicas observadas na mucosa do intestino delgado foram acúmulo de material mucoide (Fig.1A) e petéquias (Fig.1B). Em todas as análises macroscópicas não foi observado efeito da qualidade da gordura das dietas sobre as lesões. Entretanto, o desafio vacinal com Eimeria no primeiro dia de vida causou lesão mais grave no trato digestivo de frangos aos 14, 21 e 28 dias, quando comparado às aves que não receberam o desafio. Aos 35 dias de idade não foram observadas diferenças entre os tratamentos (Quadro 6).

\section{Lesões microscópicas}

As principais lesões microscópicas observadas foram fusão de vilosidades (Fig.1C), e hiperplasia de células caliciformes (Fig.1D). Aos 14 dias, a histologia da mucosa dos diferentes segmentos do intestino não teve interferência da qualidade da gordura, porém, as aves desafiadas com Eimeria no dia 1 apresentaram incremento nas lesões microscópicas no duodeno e íleo (Quadro 7).

Aos 21 dias de idade foi observada interferência da gordura na ração sobre as lesões da mucosa intestinal de frangos de corte. Aves alimentadas com gordura oxidada, desafiadas com Eimeria no dia 1 e vacinadas contra doença de Gumboro no dia 16 mostraram alto escore de lesões microscópicas em todos os segmentos do intestino delgado (Quadro 8).

Aos 28 dias de idade foi observado que aves alimentadas com gordura de baixa qualidade apresentaram le-

Quadro 5. Média e erro padrão do ganho de peso (GP) em gramas de frangos de corte nos diferentes tratamentos

\begin{tabular}{|c|c|c|c|c|c|c|}
\hline \multicolumn{2}{|c|}{ Tratamentos } & \multirow[t]{2}{*}{ GP 1-21d } & \multirow[t]{2}{*}{ GP 14-21d } & \multirow[t]{2}{*}{ GP 1-28d } & \multirow[t]{2}{*}{ GP $14-28 d$} & \multirow[t]{2}{*}{ GP $1-35 d$} \\
\hline Coccidiose & Gumboro & & & & & \\
\hline - & - & $819,17 \pm 176,53$ & $433,13 \pm 129,51 \mathrm{ab}$ & $1508,4 \pm 207,57$ & $1115,8 \pm 167,29 a$ & $2257,5 \pm 101,21$ \\
\hline Dia 1 & & $787,02 \pm 146,20$ & $407,04 \pm 120,79 b$ & $1448,3 \pm 192,92$ & $1066,8 \pm 162,87 \mathrm{ab}$ & $2142,0 \pm 279,69$ \\
\hline Dia 1 & Dia 16 & $812,96 \pm 146,30$ & $420,85 \pm 123,47 \mathrm{ab}$ & $1423,0 \pm 200,50$ & $1027,9 \pm 161,38 b$ & $2106,1 \pm 246,63$ \\
\hline \multirow[t]{2}{*}{ Dia 20} & Dia 16 & $852,21 \pm 124,68$ & $468,96 \pm 69,52 a$ & $1482,4 \pm 213,04$ & $1104,3 \pm 165,52 \mathrm{ab}$ & $2115,1 \pm 199,30$ \\
\hline & & & & Probabilidades & & \\
\hline \multirow{2}{*}{\multicolumn{2}{|c|}{ Qualidade do óleo }} & 0,052 & 0,198 & 0,055 & 0,125 & 0,068 \\
\hline & & 0,055 & 0,011 & 0,122 & 0,037 & 0,114 \\
\hline
\end{tabular}

Letras diferentes na mesma coluna apresentam diferença estatística pelo teste de Kruskal-Wallis $(\mathrm{P}<0,05)$.

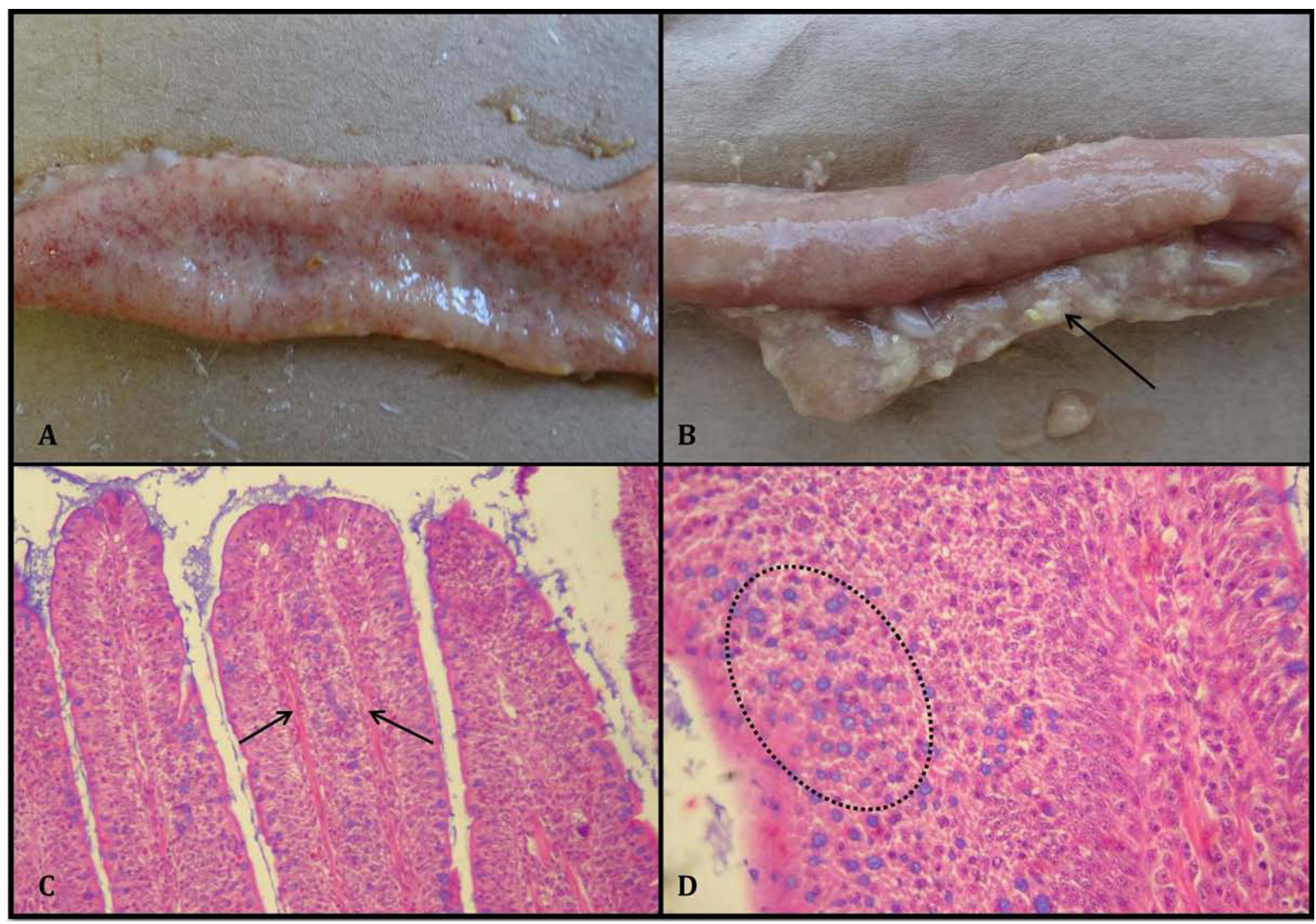

Fig.1. (A) Petéquias na mucosa do duodeno. (B) Acumulo de material mucoide na mucosa do jejuno (seta). (C) Fusão de vilosidades no jejuno (cada seta corresponde a uma vilosidade). HE, obj.20x. (D) Hiperplasia de células caliciformes em vilosidade do duodeno (área demarcada). HE, obj.40x. 
Quadro 6. Média e erro padrão do escore das lesões macroscópicas no intestino de frangos de corte dos 14 aos 35 dias de idade

\begin{tabular}{|c|c|c|c|c|c|}
\hline \multicolumn{2}{|c|}{ Tratamentos } & \multirow[t]{2}{*}{14 dias } & \multirow[t]{2}{*}{21 dias } & \multirow[t]{2}{*}{28 dias } & \multirow[t]{2}{*}{35 dias } \\
\hline Coccidiose & Gumboro & & & & \\
\hline - & - & $0,000 \pm 0,000 \mathrm{a}$ & $3,417 \pm 2,466 \mathrm{a}$ & $4,583 \pm 2,193 a$ & $5,000 \pm 2,892$ \\
\hline Dia 1 & & $2,333 \pm 2,774 b$ & $9,583 \pm 4,187 b$ & $8,500 \pm 4,982 a b$ & $6,417 \pm 3,895$ \\
\hline Dia 1 & Dia 16 & $2,750 \pm 3,596 b$ & $9,333 \pm 3,725 b$ & $10,167 \pm 3,904 b$ & $4,917 \pm 3,630$ \\
\hline \multirow[t]{2}{*}{ Dia 20} & Dia 16 & $0,000 \pm 0,000 \mathrm{a}$ & $7,083 \pm 4,441 \mathrm{ab}$ & $9,417 \pm 4,122 b$ & $6,167 \pm 3,486$ \\
\hline & & & Probabilidades & & \\
\hline \multirow{2}{*}{\multicolumn{2}{|c|}{$\begin{array}{l}\text { Qualidade do óleo } \\
\text { Desafio vacinal }\end{array}$}} & 0,509 & 0,780 & 0,918 & 0,418 \\
\hline & & 0,001 & 0,001 & 0,005 & 0,630 \\
\hline
\end{tabular}

Letras diferentes na mesma coluna apresentam diferença estatística pelo teste de Kruskal-Wallis $(\mathrm{P}<0,05)$.

\begin{abstract}
Quadro 7. Média e erro padrão do escore das lesões microscópicas observadas na mucosa intestinal de duodeno, jejuno e íleo de frangos de corte aos 14 dias de idade
\end{abstract}

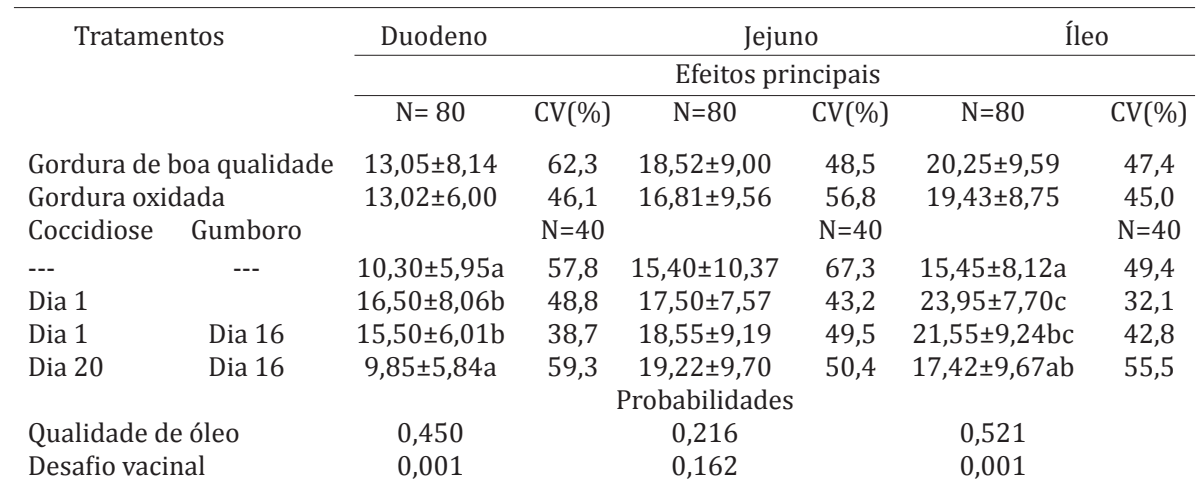

Letras diferentes na mesma coluna apresentam diferença estatística pelo teste de Kruskal-Wallis $(\mathrm{P}<0,05)$.

sões significativamente mais severas na mucosa duodenal. Frangos desafiados com vacina contra coccidiose no dia 1 e vacina contra doença de Gumboro no dia 16 apresentaram alto escore de lesões neste segmento. Neste período de 28 dias, não foi observado efeito nem da qualidade de gordura, nem dos desafios vacinais sobre o aparecimento de lesões no jejuno. No íleo, apesar de não ser observado o efeito da qualidade da gordura, o desafios com coccidiose no primeiro dia de vida das aves aumentou o escore de lesões microscópicas, comparado ao grupo não vacinado (Quadro 9).

Aos 35 dias de idade foi observada interferência da qualidade da gordura e do desafio vacinal no aparecimento de lesões na mucosa do duodeno. Frangos alimentados com gordura de baixa qualidade e submetidos aos diferentes desafios vacinais apresentaram mais lesões na mucosa do duodeno que as aves não vacinadas e que se alimentaram com gordura de boa qualidade. Na mucosa do jejuno não houve efeito da gordura de baixa qualidade. Somente aves submetidas aos desafios de coccidiose ao primeiro dia e vacina contra doença de Gumboro ao 16 dia apresentaram mais lesões no jejuno, quando comparado ao grupo de aves não vacinado. No íleo dos frangos de corte aos 35 dias foi observado apenas o efeito da qualidade de gordura, onde aves alimentadas com gordura de pior qualidade apresentaram maior escore de lesões neste segmento (Quadro 10).

\section{DISCUSSÃO}

Os resultados deste trabalho demonstraram que entre os dias 1 e 14 de idade, a qualidade do óleo e os protocolos de desafio vacinal não interferiram no peso das aves. De fato, a qualidade da gordura da dieta em nenhum momento interferiu com o ganho de peso das aves e o programa de desafio vacinal somente afetou este parâmetro no período de maior pico de resposta imunológica às Eimerias, ou seja, entre 14 e 21 dias após o desafio (Lillehoj 1998, Yun et al. 2000). Sabe-se que a vacina comercial contra coccidiose utilizada é composta de baixas doses de oocistos vivos, e foi observado que mesmo utilizando-se 10 vezes a dose recomendada pelo fabricante não houve grandes efeitos desta vacina sobre o desempenho de frangos de corte. Estes resultados podem ser comparados a outros já descritos na literatura (Williams \& Gobbi 2002, Lee Kyung-Woo et al. 2013), os quais não observaram efeito negativo no desempenho de aves vacinadas com vacinas vivas contra coccidiose em doses recomendadas pelo fabricante. Por outro lado, as dietas utilizadas no presente estudo eram à base de milho e soja e, já foi descrito, que frangos reagem menos severamente à infecção por Eimeria quando alimentados com rações à base de milho, quando comparados a frangos alimentados com ração à base de trigo (Williams 1992).

O baixo ganho de peso das aves entre os 14-28 dias está associado à maior observação de lesões macro e microscópicas, o que sugere que esteja relacionado ao ciclo de vida 
Quadro 8. Média e erro padrão do escore das lesões microscópicas observadas na mucosa intestinal do duodeno, jejuno e íleo de frangos de corte aos 21 dias de idade

\begin{tabular}{|c|c|c|c|c|c|c|c|}
\hline \multicolumn{2}{|c|}{ Tratamentos } & \multicolumn{2}{|l|}{ Duodeno } & \multicolumn{2}{|c|}{ Jejuno } & \multicolumn{2}{|l|}{ Íleo } \\
\hline & & \multicolumn{6}{|c|}{ Efeitos principais } \\
\hline & & $\mathrm{N}=80$ & $\mathrm{CV}(\%)$ & $\mathrm{N}=80$ & $\mathrm{CV}(\%)$ & $\mathrm{N}=80$ & $\mathrm{CV}(\%)$ \\
\hline \multicolumn{2}{|c|}{ Gordura de boa qualidade } & $7,63 \pm 3,09$ & 40,5 & $16,22 \pm 7,92$ & 48,8 & $16,91 \pm 9,10$ & 53,89 \\
\hline \multicolumn{2}{|c|}{ Gordura oxidada } & $13,19 \pm 4,87$ & 36,9 & $23,65 \pm 8,59$ & 36,3 & $23,64 \pm 7,85$ & 33,2 \\
\hline Coccidiose & Gumboro & $\mathrm{N}=20$ & & $\mathrm{~N}=20$ & & $\mathrm{~N}=20$ & \\
\hline--- & --- & $5,85 \pm 3,17 \mathrm{c}$ & 54,1 & $14,65 \pm 8,78 b$ & 59,9 & $13,25 \pm 7,07 c$ & 53,3 \\
\hline Dia 1 & & $9,35 \pm 2,06 \mathrm{bc}$ & 22 & $17,65 \pm 5,32 \mathrm{ab}$ & 30,1 & $19,30 \pm 9,39 a b c$ & 48,6 \\
\hline Dia 1 & Dia 16 & $9,25 \pm 3,27 b c$ & 35,4 & $15,05 \pm 9,80 b$ & 65,1 & $15,25 \pm 6,59 b c$ & 43,2 \\
\hline Dia 20 & Dia 16 & $6,10 \pm 1,77 c$ & 29,1 & $17,55 \pm 7,15 \mathrm{ab}$ & 40,7 & $19,85 \pm 11,41 \mathrm{abc}$ & 57,5 \\
\hline --- & --- & $9,35 \pm 3,90 \mathrm{bc}$ & 41,6 & $21,95 \pm 10,78 a b$ & 49,1 & $23,25 \pm 8,50 \mathrm{ab}$ & 36,6 \\
\hline Dia 1 & & $12,00 \pm 4,41 \mathrm{ab}$ & 36,8 & $24,15 \pm 7,95 a$ & 32,9 & $25,05 \pm 6,82 a$ & 27,24 \\
\hline Dia 1 & Dia 16 & $17,25 \pm 2,63 a$ & 15,26 & $26,35 \pm 4,92 \mathrm{a}$ & 18,7 & $26,05 \pm 6,52 \mathrm{a}$ & 25 \\
\hline \multirow[t]{2}{*}{ Dia 20} & Dia 16 & $14,15 \pm 4,67 \mathrm{ab}$ & 33 & $22,15 \pm 9,47 \mathrm{ab}$ & 42,7 & $20,20 \pm 8,59 a b c$ & 42,5 \\
\hline & & & & Probabilidades & & & \\
\hline \multirow{2}{*}{\multicolumn{2}{|c|}{$\begin{array}{l}\text { Qualidade do óleo } \\
\text { Desafio vacinal }\end{array}$}} & 0,001 & & 0,001 & & 0,001 & \\
\hline & & 0,001 & & 0,001 & & 0,001 & \\
\hline
\end{tabular}

Letras diferentes na mesma coluna apresentam diferença estatística pelo teste de Kruskal-Wallis $(\mathrm{P}<0,05)$.

Quadro 9. Média e erro padrão do escore das lesões microscópicas observadas na mucosa intestinal do duodeno, jejuno e íleo de frangos de corte aos 28 dias de idade

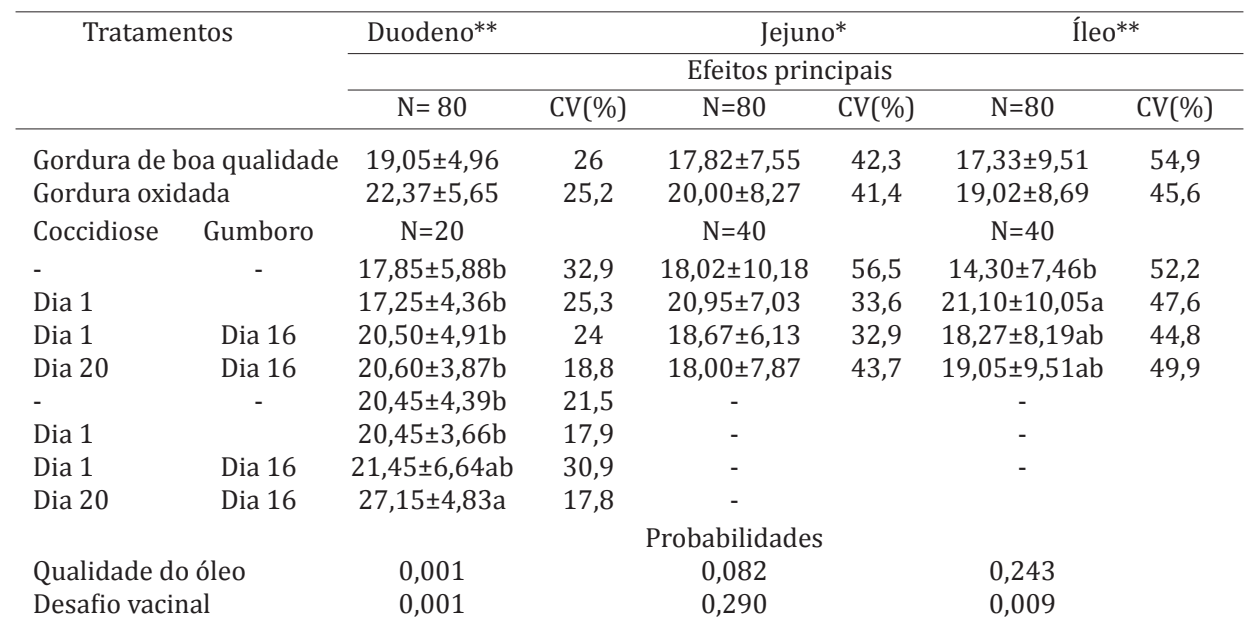

Letras diferentes na mesma coluna apresentam diferença estatística pelo teste de Kruskal-Wallis (**) e Tukey $\left({ }^{*}\right)(\mathrm{P}<0,05)$.

do parasita e à resposta do hospedeiro. Estes eventos incluem tanto o desenvolvimento endógeno no hospedeiro, como a resposta inflamatória que podem causar lesões na mucosa intestinal (Chapman 2000). Este resultado está em concordância com Williams \& Andrews (2001), que evidenciaram que lesões intestinais decorrentes da vacinação contra coccidiose podem ocorrer entre 5 e 23 dias após a vacinação, resultando em descamação, inflamação, atraso no crescimento e pior conversão alimentar (Répérant et al. 2012).

0 efeito do programa desafio vacinal sobre os parâmetros avaliados torna-se mais evidente no período de 21-28 dias de idade, quando a vacina com estirpe intermediária contra a doença de Gumboro é aplicada em um grupo de aves. Esses achados podem ser justificados pela resposta inflamatória aguda, que de acordo com Klasing (2007), aumenta a síntese de proteínas de fase aguda, diminui o apetite das aves, causa redistribuição de nutrientes no or- ganismo, de alguns aminoácidos como a lisina, e aumentam o custo da resposta imunológica. Estima-se que a resposta imunológica aguda possa utilizar $550 \mu \mathrm{mol} / \mathrm{kg} /$ dia de lisina, porém, se essa quantidade de lisina fosse utilizada para o crescimento poderia se obter 7,8 g de massa corporal $/ \mathrm{kg}$ de peso vivo (Klasing 2004).

Nas análises histopatológicas do intestino aos 21 dias de idade foi evidenciado que as aves alimentadas com gordura de baixa qualidade na ração apresentavam maior escore de lesões. Tem se demonstrado que estes tipos de gorduras podem gerar prejuízos nos tecidos e diminuir o desempenho das aves (Lin et al. 1989, Tavárez et al. 2011). Basicamente, estas gorduras conduzem a um estado de estresse oxidativo, que ocorre quando a formação ou ingestão de oxidantes excede a capacidade do sistema antioxidante de remover estes radicais livres ou espécies reativas de oxigênio (ERO) (Urso \& Clarkson 2003). Sob estas condições, as células são submetidas a um ataque direto por ERO, e 
Quadro 10. Média e erro padrão do escore das lesões microscópicas observadas na mucosa do duodeno, jejuno e íleo de frangos de corte aos 35 dias de idade

\begin{tabular}{|c|c|c|c|c|c|c|c|}
\hline \multirow{3}{*}{\multicolumn{2}{|c|}{ Tratamentos }} & \multirow{2}{*}{\multicolumn{4}{|c|}{$\begin{array}{c}\text { Jejuno* } \\
\text { Efeitos principais } \\
\end{array}$}} & \multicolumn{2}{|c|}{ Íleo* } \\
\hline & & & & & & & \\
\hline & & $N=80$ & $\mathrm{CV}(\%)$ & $\mathrm{N}=80$ & CV(\%) & $\mathrm{N}=80$ & $\mathrm{CV}(\%)$ \\
\hline \multirow{2}{*}{\multicolumn{2}{|c|}{$\begin{array}{l}\text { Gordura de boa qualidade } \\
\text { Gordura oxidada }\end{array}$}} & $18,30 \pm 5,90$ & 32,3 & $18,32 \pm 7,66$ & 41,8 & $19,19 \pm 8,75$ & 45,6 \\
\hline & & $25,07 \pm 5,71$ & 22,8 & $20,80 \pm 8,64$ & 41,5 & $22,05 \pm 8,34$ & 37,8 \\
\hline \multicolumn{2}{|c|}{ Coccidiose Gumboro } & $\mathrm{N}=20$ & & $\mathrm{~N}=40$ & & $\mathrm{~N}=20$ & \\
\hline- & - & $18,15 \pm 5,25 c$ & 28,9 & $16,82 \pm 8,23 b$ & 37,4 & $17,65 \pm 5,82$ & 32,9 \\
\hline Dia 1 & & $19,20 \pm 4,78 b c$ & 24,9 & $20,67 \pm 8,12 a b$ & 45,1 & $18,15 \pm 10,75$ & 59,2 \\
\hline Dia 1 & Dia 16 & $16,60 \pm 5,15 c$ & 31 & $21,72 \pm 7,92 \mathrm{a}$ & 32,3 & $21,35 \pm 7,03$ & 32,9 \\
\hline Dia 20 & Dia 16 & $19,25 \pm 7,95 b c$ & 41,3 & $19,02 \pm 8,10 \mathrm{ab}$ & 52,9 & $19,60 \pm 10.52$ & 53,7 \\
\hline- & - & $19,85 \pm 4,69 \mathrm{bc}$ & 23,6 & - & & $25,75 \pm 8,19$ & 31,8 \\
\hline Dia 1 & & $26,90 \pm 4,99 a$ & 18,6 & - & & $21,75 \pm 6,61$ & 30,4 \\
\hline Dia 1 & Dia 16 & $25,30 \pm 5,19 a b$ & 20,5 & - & & $21,10 \pm 6,85$ & 32,5 \\
\hline \multirow[t]{2}{*}{ Dia 20} & Dia 16 & $28,25 \pm 4,33 a$ & 15,3 & - & & $19,60 \pm 10,47$ & 53,4 \\
\hline & & & & Probabilidades & & & \\
\hline \multirow{2}{*}{\multicolumn{2}{|c|}{$\begin{array}{l}\text { Qualidade do óleo } \\
\text { Desafio vacinal }\end{array}$}} & 0,001 & & 0,053 & & 0,035 & \\
\hline & & 0,001 & & 0,040 & & 0,100 & \\
\hline
\end{tabular}

Letras diferentes na mesma coluna apresentam diferença estatística pelo teste de Kruskal-Wallis $\left.{ }^{* *}\right)$ e Tukey $\left(^{*}\right)(\mathrm{P}<0,05)$.

significativas alterações moleculares e celulares podem ocorrer (Zhang et al. 2010). Os resultados apresentados concordam com Dibner et al. (1996), que observaram que a gordura oxidada pode ter efeitos deletérios diretos em enterócitos, como a diminuição da vida média e da absorção de nutrientes devido a mudanças na permeabilidade e atividade secretória. Finalmente, estes efeitos primários podem conduzir a efeitos sistêmicos secundários, como o baixo ganho de peso e pobre desempenho das aves.

Desta forma, conclui-se que o uso de fatores não infecciosos como a gordura de baixa qualidade e o desafio infeccioso subclínico com vacina contra coccidiose associado à vacina com uma estirpe intermediária do vírus da doença de Gumboro foi suficiente para induzir um quadro de enterite inespecífica branda em frangos de corte sob condições experimentais. Acredita-se que este protocolo seja importante para avaliar programas de vacinação e/ou aditivos que se propõe a resolver o problema no campo. 0 uso do escore de lesão aplicado para alterações macro e microscópicas possibilitou a utilização de programas de estatística para comparação dos resultados de forma clara e rápida, sendo fundamental para a compreensão dos achados. Assim, sugerimos que este protocolo de indução de enterite em frangos de corte, associado a estas análises seja aplicado em outros estudos para compreender e buscar soluções para os problemas entéricos inespecíficos em frangos de corte.

\section{REFERÊNCIAS}

Awad A., El-Nahas A. \& Abu-Akkada S. 2013. Evaluation of the protective efficacy of the anticoccidial vaccine Coccivac-B in broilers, when challenged with Egyptian field isolates of E. tenella. Parasitol. Res. 112:113121.

Bracarense A.-P., Lucioli J., Grenier B., Drociunas Pacheco G., Moll W.-D., Schatzmayr G. \& Oswald I.P. 2012. Chronic ingestion of deoxynivalenol and fumonisin, alone or in interaction, induces morphological and immunological changes in the intestine of piglets. Brit. J. Nutr. 107:17761786.

Chapman H.D. 2000. Practical use of vaccines for the control of coccidiosis in the chicken. World's Poult. Sci. J. 56:7-20.
Dänicke S., Valenta H., Ueberschär K.H. \& Matthes S. 2007. On the interactions between Fusarium toxin-contaminated wheat and non-starch-polysaccharide hydrolysing enzymes in turkey diets on performance, health and carry-over of deoxynivalenol and zearalenone. Brit. Poult. Sci. 48:39-48.

Dibner J.J., Atwell C.A., Kitchell M.L., Shermer W.D. \& Ivey FJ. 1996. Feeding of oxidized fats to broilers and swine: effects on enterocyte turnover, hepatocyte proliferation and the gut associated lymphoid tissue. Anim. Feed Sci. Technol. 62:1-13.

Havenstein G., Ferket P. \& Qureshi M. 2003. Growth, livability, and feed conversion of 1957 versus 2001 broilers when fed representative 1957 and 2001 broiler diets. Poult. Sci. 82:1500-1508.

Hetland H., Choct M. \& Svihus B. 2004. Role of insoluble non-starch polysaccharides in poultry nutrition. World's Poult. Sci. J. 60:415-422.

Hoerr F.J. 2010. Clinical aspects of immunosuppression in poultry. Avian Dis. 54:2-15.

Jang S.I., Lillehoj H.S., Lee S.-H., Lee K.W., Lillehoj E.P., Hong Y.H., An D.-J., Jeong W., Chun J.-E., Bertrand F., Dupuis L., Deville S. \& Arous J.B. 2012. Vaccination with Clostridium perfringens recombinant proteins in combination with Montanide ${ }^{\mathrm{TM}}$ ISA 71 VG adjuvant increases protection against experimental necrotic enteritis in commercial broiler chickens. Vaccine. 30:5401-5406.

Jayaraman S., Thangavel G., Kurian H., Mani R., Mukkalil R. \& Chirakkal H. 2013. Bacillus subtilis PB6 improves intestinal health of broiler chickens challenged with Clostridium perfringens-induced necrotic enteritis. Poult. Sci. 92:370-374.

Klasing K.C. 2004. The costs of immunity. Acta Zool. Sinica. 50:961-969.

Klasing K.C. 2007. Nutrition and the immune system. Brit. Poult. Sci. 48:525-537.

Lanckriet A., Timbermont L., De Gussem M., Marien M., Vancraeynest D., Haesebrouck F., Ducatelle R. \& Van Immerseel F. 2010. The effect of commonly used anticoccidials and antibiotics in a subclinical necrotic enteritis model. Avian Pathol. 39:63-68.

Lee K.-W., Lillehoj H.-S., Jang S.-I., Lee S.-H., Bautista D.A., Donald Ritter G., Lillehoj E.P. \& Siragusa G.R. 2013. Comparison of live Eimeria vaccination with in-feed salinomycin on growth and immune status in broiler chickens. Res. Vet. Sci. 95:110-114.

Lee K.W., Lee S.H., Lillehoj H.S., Li G.X., Jang S.I., Babu U.S., Park M.S., Kim D.K., Lillehoj E.P., Neumann A.P., Rehberger T.G. \& Siragusa G.R. 2010. Effects of direct-fed microbials on growth performance, gut morphometry, and immune characteristics in broiler chickens. Poult. Sci. 89:203-216.

Lillehoj H.S. 1998. Role of T lymphocytes and cytokines in coccidiosis. Int. J. Parasitol. 28:1071-1081. 
Lin C.F., Asghar A., Gray J.I., Buckley D.J., Booren A.M., Crackel R.L. \& Flegal C.J. 1989. Effects of oxidised dietary oil and antioxidant supplementation on broiler growth and meat stability. Brit. Poult. Sci. 30:855-864.

Lokaewmanee K., Yamauchi K. \& Thongwittaya N. 2012. Effects of fermented plant product on growth performance, some blood variables, carcase characteristics, and intestinal histology in broilers. Brit. Poult. Sci. 53:215-223.

Mazariegos L.A., Lukert P.D. \& Brown J. 1990. Pathogenicity and immunosuppressive properties of infectious bursal disease "Intermediate" strains. Avian Dis. 34:203-208.

McReynolds J., Byrd J., Anderson R., Moore R., Edrington T., Genovese K., Poole T., Kubena L. \& Nisbet D. 2004. Evaluation of immunosuppressants and dietary mechanisms in an experimental disease model for necrotic enteritis. Poult. Sci. 83:1948-1952.

Olkowski A.A., Wojnarowicz C., Chirino-Trejo M., Laarveld B. \& Sawicki G. 2008. Sub-clinical necrotic enteritis in broiler chickens: Novel etiological consideration based on ultra-structural and molecular changes in the intestinal tissue. Res. Vet. Sci. 85:543-553.

Patricio I., Mendes A., Ramos A. \& Pereira D. 2012. Overview on the performance of Brazilian broilers (1990 to 2009). Rev. Bras. Cienc. Avic. 14:233-238.

Rambozzi L., Renna M., Cornale P., Perona G., Malfatto V. \& Mimosi A. 2012. Effect of the granulometric characteristics of monensin sodium on controlling experimental coccidiosis in broiler chickens. Rev. Bras. Parasitol. Vet. 21:60-64

Répérant J.-M., Dardi M., Pagès M. \& Thomas-Hénaff M. 2012. Pathogenicity of Eimeria praecox alone or associated with Eimeria acervulina in experimentally infected broiler chickens. Vet. Parasitol. 187:333-336.

Rostagno H.S. 2011. Tabelas Brasileiras para aves e suínos. 3 ed. Universidade Federal de Viçosa, Viçosa. 252p.

Simitzis P.E., Kalogeraki E., Goliomytis M., Charismiadou M.A., Triantaphyllopoulos K., Ayoutanti A., Niforou K., Hager-Theodorides A.L. \& Deligeorgis S.G. 2012. Impact of stocking density on broiler growth performance, meat characteristics, behavioural components and indicators of physiological and oxidative stress. Brit. Poult. Sci. 53:721-730.

Tavárez M.A., Boler D.D., Bess K.N., Zhao J., Yan F., Dilger A.C., McKeith F.K. \& Killefer J. 2011. Effect of antioxidant inclusion and oil quality on broiler performance, meat quality, and lipid oxidation. Poult. Sci. 90:922-930.
Teirlynck E., Gussem M.D.E., Dewulf J., Haesebrouck F., Ducatelle R. \& Van Immerseel F. 2011. Morphometric evaluation of "dysbacteriosis" in broilers. Avian Pathol. 40:139-144.

Teirlynck E., Bjerrum L., Eeckhaut V., Huygebaert G., Pasmans F., Haesebrouck F., Dewulf J., Ducatelle R. \& Van Immerseel F. 2009. The cereal type in feed influences gut wall morphology and intestinal immune cell infiltration in broiler chickens. Brit. J. Nutr. 102:1453-1461.

Timbermont L., Haesebrouck F., Ducatelle R. \& Van Immerseel F. 2011. Necrotic enteritis in broilers: an updated review on the pathogenesis. Avian Pathol. 40:341-347.

Tolkamp B., Wall E., Roehe R., Newbold J. \& Zaralis K. 2010. Review of nutrient efficiency in different breeds of farm livestock. Department for Environment, Food \& Rural Affairs, London. 105p.

Urso M.L. \& Clarkson P.M. 2003. Oxidative stress, exercise, and antioxidant supplementation. Toxicol. 189:41-54.

Williams R.B. 1992. Differences between the anticoccidial potencies of monensin in maize-based or wheat-based chicken diets. Vet. Res. Commun. 16:147-152.

Williams R.B. 2005. Intercurrent coccidiosis and necrotic enteritis of chickens: rational, integrated disease management by maintenance of gut integrity. Avian Pathol. 34:159-180.

Williams R.B. \& Andrews S.J. 2001. The origins and biological significance of the coccidial lesions that occur in chickens vaccinated with a live attenuated anticoccidial vaccine. Avian Pathol. 30:215-220.

Williams R.B. \& Gobbi L. 2002. Comparison of an attenuated anticoccidial vaccine and an anticoccidial drug programme in commercial broiler chickens in Italy. Avian Pathol. 31:253-265.

Yun C.H., Lillehoj H.S. \& Lillehoj E.P. 2000. Intestinal immune responses to coccidiosis. Dev. Comp. Immunol. 24:303-324.

Zekarias B., Stockhofe-Zurwieden N., Post J., Balk F., van Reenen C., Gruys E. \& Rebel J.M.J. 2005. The pathogenesis of and susceptibility to malabsorption syndrome in broilers is associated with heterophil influx into the intestinal mucosa and epithelial apoptosis. Avian Pathol. 34:402407.

Zhang W., Xiao S., Lee E.J. \& Ahn D.U. 2010. Consumption of oxidized oil increases oxidative stress in broilers and affects the quality of breast meat. J. Agric. Food Chem. 59:969-974. 